\title{
New actionable targets and investigational drugs in chronic lymphocytic leukemia
}

\author{
Jan-Paul Bohn (iD)
}

Received: 16 August 2021 / Accepted: 2 September 2021 / Published online: 5 October 2021

(C) The Author(s) 2021

\begin{abstract}
Summary The treatment landscape of chronic lymphocytic leukemia (CLL) has shifted from chemotherapy-based approaches to targeted agents in the last decade. However, evolving drug resistance and accumulating toxicity remain challenges that still limit patients' clinical outcomes. Furthermore, currently licensed targeted agents such as inhibitors of Bruton's tyrosine kinase (BTK) and anti-apoptotic protein B-cell lymphoma 2 (BCL2) do not adequately compensate for the poor clinical outcomes associated with high-risk genetics such as TP53 alterations. New insights into disease biology facilitated design and investigation of several new targeted agents with encouraging results in early clinical trials. This short review focuses on novel actionable targets and investigational drugs aimed at circumventing acquired resistance and avoiding accumulating toxicity.
\end{abstract}

Keywords Pirtobrutinib - Umbralisib - Tafasitamab · CAR-T cell immunotherapy $\cdot$ Lisocabtagene maraleucel

\section{Introduction}

The treatment landscape of chronic lymphocytic leukemia (CLL) has undergone profound change in the last decade. From chemotherapy-based regimens accomplishing first overall survival benefits in firstline therapy [1], treatment-associated toxicity and inferior clinical outcomes in patients with high-risk disease biology necessitated alternative treatment approaches with targeted agents. Superior in terms of efficacy and tolerability, inhibitors of Bruton's tyrosine

J.-P. Bohn, M.D., PhD (ه)

Department of Internal Medicine V, Medical University of Innsbruck, Anichstr. 35, 6020 Innsbruck, Austria

jan-paul.bohn@i-med.ac.at kinase (BTK) and anti-apoptotic protein B-cell lymphoma 2 (BCL2) entered the arena of CLL therapy in record time and strongly shifted chemoimmunotherapy into the background of commonly accepted treatment algorithms [2-5]. However, acquired resistance and drug-associated toxicity remain a persisting issue with currently licensed CLL therapeutics rendering most patients to several lines of treatment throughout the course of the disease [6, 7]. In particular, high-risk genetics such as unmutated immunoglobulin heavy chain variant region (IGHV) status, TP53 alterations and a complex karyotype continue to pose therapeutic challenges and are associated with a more aggressive course of disease and poor clinical outcomes [3,4]. New insights into disease biology initiated the investigation of several new potential therapeutic targets with promising results in early clinical trials. This review focuses on novel investigational drugs aimed at circumventing emerging drugresistance and avoiding accumulating toxicity.

\section{Reversible Bruton's tyrosine kinase inhibitors}

BTK inhibition has proceeded to become an integral part of CLL treatment [8]. Positioned directly downstream of the constitutively activated B-cell receptor in CLL cells, inhibition of BTK hampers multiple cellular processes, including cell proliferation and migration. As such, the malignant cells are deprived from the nourishing stimuli of their local microenvironment and more easily succumb to apoptosis [9]. Nevertheless, minimal residual disease (MRD) frequently persists with monotherapy requiring continuous treatment to allow long-term disease control [10]. Accordingly, acquired resistance commonly evolves and frequently involves gene mutations altering the binding site Cys481 of currently licensed irreversible BTK inhibitors such as ibrutinib and acalabrutinib [11, 
12]. At ASCO 2021, early results of ELEVATE-RR [13], a randomized phase 3 study comparing acalabrutinib ( $n=268)$ head-to-head with ibrutinib $(n=265)$ in relapsed CLL patients harboring either a deletion of $17 p$ or $11 q$, were presented, meeting its primary endpoint of non-inferiority progression-free survival (PFS, 38.4 months, hazard ratio 1.00) with a median follow-up of 41 months and demonstrating a favorable toxicity profile of acalabrutinib in terms of atrial fibrillation (9\% vs. 15.6\%), low-grade bleeding events (38\% vs. $51.3 \%$ ) and hypertension (9.4\% vs. $23.2 \%)$ [13]. Zanubrutinib, another irreversible BTK inhibitor still under investigation in CLL, has also been compared with ibrutinib in the randomized phase 3 trial ALPINE in 415 relapsed CLL patients. With a median follow-up of only 14 months, very early data indicate a PFS benefit with zanubrutinib at 1 year $(94.9 \%$ vs. $84.0 \%, p=0.0007$ ) and reduced incidence of any grade atrial fibrillation (2.5\% vs. $10.1 \%$ ) [14].

To overcome acquired resistance, reversible BTK inhibitors have been designed to inhibit BTK by noncovalent binding. Following encouraging in vitro results demonstrating effective blockade of BTK signaling in ibrutinib-resistant BTK C481S mutants, several compounds have entered early clinical investigation [15-17]. Pirtobrutinib (LOXO-305) is currently among the most promising and highly selective compounds of these non-covalent binding next generation BTK inhibitors [18]. In an ongoing phase $1 / 2$ trial in 323 patients with relapsed/refractory B cell malignancies no dose-limiting toxicity was observed leading to the phase 2 dose of $200 \mathrm{mg}$ daily. The most common adverse events (AE) were fatigue $(20 \%)$, diarrhea $(17 \%)$, and contusion (13\%). Notably, grade $3 / 4$ atrial fibrillation was not seen. All 170 CLL patients included were previously treated with an irreversible BTK inhibitor and received a median of four preceding lines of treatment. Overall response rate (ORR) was $62 \%$ and similar between CLL patients intolerant or resistant to previous covalent BTK inhibition $(52 \%$ versus $67 \%$ ) [18].

Taken together, these early data suggest that pirtobrutinib is active and well-tolerated in multiple pretreated CLL patients, including those resistant or intolerant to irreversible BTK inhibitors. Upcoming randomized controlled clinical trials, however, are eagerly awaited to better define the role of this new class of drugs in the treatment landscape of CLL (NCT04666038, NCT04965493).

\section{Next-generation PI3K inhibitors}

Besides chronic activation of BTK, aberrant B-cell receptor stimulation in CLL leads to constitutive PI3K signaling essential for B-cell proliferation and survival [19].

In 2014, the first-in-class inhibitor of the PI3K subunit delta idelalisib was approved for relapsed, refractory CLL patients by the US Food and Drug Admin- istration (FDA) and the European Medicines Agency (EMA) [20]. However, accumulating immunogenic AE including hepatotoxicity, colitis, and pneumonitis as well as infectious complications, such as pneumocystis jirovecii pneumonia and cytomegalovirus reactivation, resulted in a black box warning and premature discontinuation of multiple ongoing clinical trials [21]. Hence, idelalisib is often reserved for patients resistant or intolerant to inhibitors of BTK and BCL2, but remains an effective treatment option in relapsed/ refractory CLL.

Umbralisib is a structurally distinct next-generation PI3K inhibitor with a greater selectivity for the delta subunit. Contrary to idelalisib, umbralisib has not been associated with immunogenic AE. Most common AE were low-grade and included diarrhea $(43 \%)$, nausea $(42 \%)$, and fatigue $(31 \%)$ when used as monotherapy [22]. At ASH 2020, first results from a phase 3 study were presented comparing umbralisib combined with the novel anti-CD20 antibody ublituximab versus chlorambucil and obinutuzumab in 421 CLL patients (untreated $n=240$, relapsed/refractory $n=181$ ) [23]. ORR was significantly higher in the experimental study arm (83.3\% vs. $68.7 \%, p<0.001)$, while the benefit was mainly derived from the previously treated study population ( $82 \%$ vs. $57 \%$ ORR), in particular those patients failing previous BTK inhibitor treatment ( $57 \%$ vs. $25 \%$ ORR). At a median follow-up of 36.2 months, progression-free survival (PFS) was significantly prolonged with umbralisib and ublituximab versus chemoimmunotherapy, an effect that was consistent in both treatment-naïve and relapsed/refractory CLL patients (38.5 vs. 26.1 months and 19.5 vs. 12.9 months, respectively). Data on MRD monitoring remain to be published. Grade 3/4AE of interest in both cohorts included neutropenia $(30.6 \%$ vs. $34.7 \%$ ), diarrhea ( 12.1 vs. $2.5 \%$ ), transaminitis ( $8.3 \%$ vs. $2 \%)$, colitis $(3.4 \%$ vs. $0 \%$ ) and pneumonitis (2.9\% vs. $0 \%$ ) [23].

Reviewing the feasible toxicity profile and significantly prolonged PFS versus chemoimmunotherapy suggests umbralisib plus ublituximab as a valuable addition to the CLL treatment landscape.

\section{Anti-CD19 monoclonal antibodies}

Tafasitamab is an anti-CD19 monoclonal antibody with encouraging efficacy (ORR 67\% according to clinical criteria) and adequate tolerability in a phase I trial with 27 multiple relapsed CLL patients. Most frequent AEs were low-grade infusion-related reactions (IRR) [24]. In 2019, the first results of combination treatment with either the PI3K inhibitor idelalisib $(n=11)$ or venetoclax $(n=11)$ were reported in CLL patients failing prior BTK therapy. In combination with idelalisib, ORR was $90.9 \%$ (CR $=9.1 \%$, PR $81.8 \%$ ), while one out of eight patients assessed for MRD achieved MRD negativity in the bone marrow. Most common AE comprised usually low-grade myelotox- 
icity, foremost anemia (55\%), IRR, and pyrexia (46\% each). Serious AE were reported in 8 patients, including, pulmonary infection (5 patients).

In combination with venetoclax, ORR was $76.9 \%$ (CR $=23.1 \%$, PR 53.8\%), while response was not evaluable in $23.1 \%$ of cases. Six out of 7 patients assessed for MRD in the peripheral blood were negative within 7 cycles. Most common AE included myelotoxicity, foremost high-grade neutropenia, and low-grade IRR. Serious $\mathrm{AE}$ were reported in 9 patients, including pyrexia $(n=3)$ and IRR $(n=2)$ [25].

Combination treatment with tafasitamab and lenalidomide was investigated in another phase 2 study in 25 CLL patients (untreated $n=12$, relapsed/refractory $n=13)$ [26].

Combination treatment yielded an ORR of $67 \%$ (1 CR, 7 PR) and 15\% (PR only) in treatment-naïve and relapsed/refractory CLL patients, respectively. Grade 3-4AE included pulmonary infections $(n=4)$, neutropenia $(n=3)$, and IRR $(n=2)$ in untreated patients and primarily infections $(n=7)$ in relapsed/ refractory patients [26]. These early results suggest adequate tolerability and encouraging efficacy of tafasitamab in combination with other targeted agents and warrant further investigation in larger randomized clinical trials.

\section{CAR-T cell immunotherapy}

Chimeric antigen receptor-engineered (CAR)-T cell therapy has emerged as the most complex immunotherapeutic approach in recent years, primarily directed against CD19+ B-cell malignancies [27]. CARs are genetically engineered synthetic constructs transfected into immunocompetent $\mathrm{T}$ cells of the patients to recognize a specific antigen on the surface of tumor cells [27]. Several clinical trials have demonstrated efficacy of CD19-directed CART-cell therapy leading to FDA and EMA approval in certain B-cell lymphomas [28, 29]. Early studies in CLL suggested only minor efficacy compared to B-cell acute lymphoblastic leukemia and diffuse large B-cell lymphoma with low CR rates (20-30\%) and limited PFS of $25 \%$ at 18 months [30-32], which has been attributed to the disease characteristic exhausted phenotype of CD4+ and CD8+ T cells with low proliferative and cytotoxic capacities [33]. However, preconditioning lymphodepletion with fludarabine and cyclophosphamide as well as pre- and concomitant treatment with ibrutinib may significantly enhance clinical outcomes [32, 34]. Recently, the phase I study TRANSCEND CLL 004 was updated investigating lisocabtagene maraleucel (liso-cel) either alone $(n=23)$ or in combination with ibrutinib $(n=19)$ in heavily pretreated CLL relapsed/refractory patients [35, 36]. Most patients were defined highrisk with a median of five pretreatments, including ibrutinib in all patients. In the monotherapy cohort, ORR was $82 \%$ (CR with or without incomplete count recovery (CRi) 46\%; PR 36\%) with a median PFS of
18 months. Notably, 75\% (15/20) of patients achieved MRD negativity, mostly within 30 days of therapy (60\%). Common grade 3/4AE included myelotoxicity, foremost anemia (74\%). Cytokine release syndromes were usually low-grade, observed in $74 \%$ of patients and generally manageable with corticosteroids and tocilizumab [35]. Of 19 patients beyond 1 month of follow-up in the combination treatment cohort, ORR and CR/CRi were $95 \%$ and $47 \%$, respectively. $89 \%$ of patients achieved MRD negativity in the peripheral blood as measured by flow cytometry. Common grade 3/4AE included myelotoxicity, foremost neutropenia (89\%). Cytokine release syndromes were usually lowgrade, observed in $74 \%$ of patients and generally manageable with corticosteroids and tocilizumab [36].

In summary, these early results suggest adequate tolerability and favorable efficacy of liso-cel in combination with ibrutinib in highly pretreated CLL patients. Longer follow-up of the monotherapy cohort suggests sustained deep responses at 24 months and no delayed safety signals.

With rapidly growing efficacy data and clinical experience in managing the associated toxicity profile, CAR-T cell therapy may emerge as an increasingly important treatment option for CLL patients with highrisk disease and relapsed/refractory to prior therapy with BTK- and BCL2-inhibitors.

\section{Conclusion}

In recent years, targeted therapies have revolutionized CLL (chronic lymphocytic leukemia) treatment. Foremost, inhibitors of BTK (Bruton's tyrosine kinase) and BLC2 (anti-apoptotic protein B-cell lymphoma 2) such as ibrutinib and venetoclax with or without the addition of an anti-CD20 antibody have become frontline treatment of choice regardless of patient age, comorbidity and most genetic risk factors. However, neither inhibitors of BTK nor BCL2 may overcome the inferior prognosis associated with high-risk genetics such as TP53 dysfunction and unmutated IGHV (immunoglobulin heavy chain variant region) status.

Early clinical trials currently focus on next-generation agents of established drug targets or new actionable targets with encouraging efficacy and safety. Longer follow-up and randomized clinical trials provided, some of these new compounds may indeed serve as a valuable addition to the therapeutic armamentarium in CLL. However, for now CLL remains an incurable disease and the most effective CLL treatment in the clinical and experimental setting may comprise combined therapy rather than monotherapy. Besides looking for effective and well-tolerated novel agents directed against new actionable targets, studies are therefore also warranted to analyze the best combinations and sequences of available drugs to further improve clinical outcomes in a chronic B-cell malignancy commonly requiring multiple retreatments during the course of the disease. 
Funding Open access funding provided by University of Innsbruck and Medical University of Innsbruck.

Conflict of interest J.-P. Bohn reports personal fees from AbbVie, Astra-Zeneca and Janssen for advisory board participation.

Open Access This article is licensed under a Creative Commons Attribution 4.0 International License, which permits use, sharing, adaptation, distribution and reproduction in any medium or format, as long as you give appropriate credit to the original author(s) and the source, provide a link to the Creative Commons licence, and indicate if changes were made. The images or other third party material in this article are included in the article's Creative Commons licence, unless indicated otherwise in a credit line to the material. If material is not included in the article's Creative Commons licence and your intended use is not permitted by statutory regulation or exceeds the permitted use, you will need to obtain permission directly from the copyright holder. To view a copy of this licence, visit http://creativecommons.org/licenses/by/4.0/.

\section{References}

1. Hallek M, Fischer K, Fingerle-Rowson G, et al. Addition of rituximab to fludarabine and cyclophosphamide in patients with chronic lymphocytic leukaemia: a randomised, open-label, phase 3 trial. Lancet. 2010;376:1164-74.

2. Burger JA, Tedeschi A, Barr PM, et al. Ibrutinib as initial therapy for patients with chronic lymphocytic leukemia. NEngl J Med. 2015;373:2425-37.

3. Woyach JA, Ruppert AS, Heerema NA, et al. Ibrutinib regimens versus chemoimmunotherapy in older patients with untreated CLL. NEngl J Med. 2018;379:2517-28.

4. Fischer K, Al-Sawaf O, Bahlo J, et al. Venetoclax and obinutuzumab in patients with CLL and coexisting conditions. NEngl J Med. 2019;380:2225-36.

5. ShanafeltTD, WangXV, Kay NE, et al. Ibrutinib-rituximab or chemoimmunotherapy for chronic lymphocytic leukemia. NEngl J Med. 2019;381:432-43.

6. MatoAR, NabhanC,ThompsonMC, etal. Toxicities and outcomes of 616 ibrutinib-treated patients in the United States: a real-world analysis. Haematologica. 2018;103:874-9.

7. Mato AR, Thompson M, Allan JN, et al. Real-world outcomes and management strategies for venetoclax-treated chroniclymphocyticleukemiapatientsin the United States. Haematologica. 2018;103:1511-7.

8. Eichhorst B, Robak T, Montserrat E, et al. Chronic lymphocytic leukaemia: ESMO clinical practice guidelines for diagnosis, treatment and follow-up. Ann Oncol. 2021;32:23-33.

9. Hendriks RW, Yuvaraj S, Kil LP. Targeting Bruton's tyrosine kinase in B cell malignancies. Nat Rev Cancer. 2014;14:219-32.

10. Burger JA, Barr PM, Robak T, et al. Long-term efficacy and safety of first-line ibrutinib treatment for patients with CLL/SLL: 5 years of follow-up from the phase 3 RESONATE2 study. Leukemia. 2020;34:787-98.

11. Woyach JA, Furman RR, Liu T-M, et al. Resistance mechanisms for the Bruton's tyrosine kinase inhibitor ibrutinib. NEngl J Med. 2014;370:2286-94.

12. Sharman JP, Egyed M, Jurczak W, et al. Acalabrutinib with or without obinutuzumab versus chlorambucil and obinutuzumab for treatment-naive chronic lymphocytic leukaemia (ELEVATE-TN): a randomised, controlled, phase 3 trial. Lancet. 2020;395:1278-91.

13. Byrd JC, Hillmen P, Ghia P, et al. First results of a headto-head trial of acalabrutinib versus ibrutinib in previ- ously treated chronic lymphocytic leukemia. J Clin Oncol. 2021;39:7500.

14. Hillmen P, Brown JR, Byrd JC, et al. Alpine: phase 3 trial of zanubrutinib (BGB-3111) vs ibrutinib in patients with relapsed/refractory $(\mathrm{R} / \mathrm{R})$ chronic lymphocytic leukemia/small lymphocyticlymphoma (CLL/SLL). Blood. 2019;134:4307.

15. Byrd JC, Smith S, Wagner-Johnston N, et al. First-inhuman phase 1 study of the BTK inhibitor GDC-0853 in relapsed or refractory B-cell NHL and CLL. Oncotarget. 2018;9:13023-35.

16. WoyachJ, Stephens DM, FlinnIW, etal. Final results of phase 1 , dose escalation study evaluating ARQ 531 in patients with relapsed or refractory B-cell lymphoid malignancies. Blood. 2019;134:4298-4298.

17. Allan JN, Patel K, Mato AR, et al. Ongoing results of a phase $1 \mathrm{~B} / 2$ dose-escalation and cohort-expansion study of the selective, noncovalent, reversible Bruton's tyrosine kinase inhibitor, vecabrutinib, in B-cell malignancies. Blood. 2019;134:3041.

18. Mato AR, Flinn IW, Pagel JM, et al. Results from a firstin-human, proof-of-concept phase 1 trial in pretreated B-cell malignancies for Loxo-305, a next-generation, highly selective, non-covalent BTKinhibitor. Blood. 2019;134:501.

19. Lannutti BJ, Meadows SA, Herman SEM, et al. CAL-101, a p1108 selective phosphatidylinositol-3-kinase inhibitor for the treatment of B-cell malignancies, inhibits PI3K signaling and cellular viability. Blood. 2011;117:591-4.

20. Furman RR, Sharman JP, Coutre SE, et al. Idelalisib and rituximab in relapsed chronic lymphocytic leukemia. NEngl J Med. 2014;370:997-1007.

21. Hanlon A, Brander DM. Managing toxicities of phosphatidylinositol-3-kinase (PI3K) inhibitors. Hematology Am Soc Hematol Educ Program. 2020;2020:346-56.

22. Burris HA 3rd, Flinn IW, Patel MR, et al. Umbralisib,

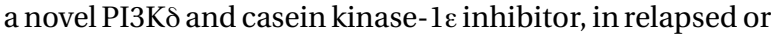
refractory chronic lymphocytic leukaemia and lymphoma: an open-label, phase 1, dose-escalation, first-in-human study. Lancet Oncol. 2018;19:486-96.

23. Gribben JG, Jurczak W, Jacobs RW, et al. Umbralisib plus ublituximab (U2) is superior to obinutuzumab plus chlorambucil $(\mathrm{O}+\mathrm{Chl})$ in patients with treatment naïve (TN) and relapsed/refractory (R/R) chronic lymphocytic leukemia (CLL): results from the phase 3 unity-CLL study. Blood. 2020;136:37-9.

24. Woyach JA, Awan F, Flinn IW, et al. A phase 1 trial of the Fc-engineered CD19 antibody XmAb5574 (MOR00208) demonstrates safety and preliminary efficacy in relapsed CLL. Blood. 2014;124:3553-60.

25. Staber PB, Jurczak W, Brugger W, et al. Primary analysis of anti-CD19 tafasitamab (MOR208) treatment in combination with idelalisib or venetoclax in R/R CLL patients who failed prior BTK inhibitor therapy (COSMOS trial). Blood. 2019;134:1754-1754.

26. Bhat SA, Huang Y, Ruppert AS, et al. Final results of a phase II study of Fc engineered, CD19 antibody tafasitamab in combination with lenalidomide or ibrutinib in patients with chronic lymphocytic leukemia (CLL). Blood. 2020;136:22-3.

27. Yin Z, Zhang Y, Wang X. Advances in chimeric antigen receptor T-cell therapy for B-cell non-Hodgkin lymphoma. BiomarkRes. 2021;9:58.

28. Schuster SJ, Bishop MR, Tam CS, et al. Tisagenlecleucel in adult relapsed or refractory diffuse large B-cell lymphoma. NEngl J Med. 2019;380:45-56.

29. Locke FL, Ghobadi A, Jacobson CA, et al. Long-term safety and activity of axicabtagene ciloleucel in refractory large 
B-cell lymphoma (ZUMA-1): a single-arm, multicentre, phase 1-2 trial. Lancet Oncol. 2019;20:31-42.

30. Turtle CJ, Hay KA, Hanafi LA, et al. Durable molecular remissions in chronic lymphocytic leukemia treated with CD19-specific chimeric antigen receptor-modified T cells after failure of ibrutinib. JClin Oncol. 2017;35:3010-20.

31. Porter DL, Hwang WT, Frey NV, et al. Chimeric antigen receptor $\mathrm{T}$ cells persist and induce sustained remissions in relapsed refractory chronic lymphocytic leukemia. Sci Transl Med. 2015;7:303ra139.

32. Fraietta JA, Lacey SF, Orlando EJ, et al. Determinants of response and resistance to $\mathrm{CD} 19$ chimeric antigen receptor (CAR) T cell therapy of chronic lymphocytic leukemia. Nat Med. 2018;24:563-71.

33. van Bruggen JAC, Martens AWJ, Fraietta JA, et al. Chronic lymphocytic leukemia cells impair mitochondrial fitness in CD8(+) T cells and impede CAR T-cell efficacy. Blood. 2019;134:44-58.

34. Sommermeyer D, Hill T, Shamah SM, et al. Fully human CD19-specificchimericantigen receptorsforT-cell therapy. Leukemia. 2017;31:2191-9.

35. Siddiqi T, SoumeraiJD, DorritieKA, et al. Updated follow-up of patients with relapsed/refractory chronic lymphocytic leukemia/small lymphocytic lymphoma treated with lisocabtagene maraleucel in the phase 1 monotherapy cohort of transcend CLL 004, including high-risk and ibrutinibtreated patients. Blood. 2020;136:40-1.

36. Wierda WG, Dorritie KA, Munoz J, et al. Transcend CLL 004: phase 1 cohort of lisocabtagene maraleucel (liso-cel) in combination with ibrutinib for patients with relapsed/ refractory (R/R) chronic lymphocytic leukemia/small lymphocyticlymphoma (CLL/SLL). Blood. 2020;136:39-40.

Publisher's Note Springer Nature remains neutral with regard to jurisdictional claims in published maps and institutional affiliations.

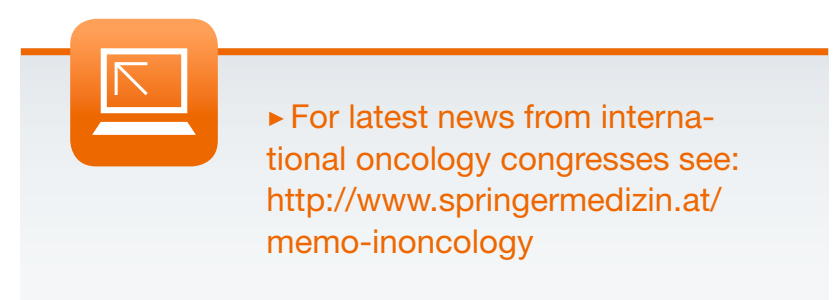

